\section{MS3-P2 Two-dimensional protein crystallography at FELs}

Cecilia M. Casadei ${ }^{1}$, Bill Pedrini ${ }^{1}$

\section{Paul Scherrer Institute}

email: cecilia.casadei@psi.ch

Membrane proteins are involved in a number of crucial and diverse biological functions ranging from cross-membrane transportation to signal reception. The structural characterization of these macromolecules is hindered by the difficulty in producing well diffracting three-dimensional crystals, limiting the range of application of conventional X-ray crystallography in these systems.

Membrane proteins arrange favorably in periodic monolayers in a lipidic environment. This configuration presents the advantage that molecules are found in a close-to-physiological arrangement. In addition, the use of ultrashort free electron laser (FEL) X-ray pulses allows to largely outrun radiation damage phenomena in biological samples at ambient temperature.

Two-dimensional (2D) protein crystals of bacteriorhodopsin proved to give rise to diffraction spots to resolution of $4 \AA$ at the Linac Coherent Light Source FEL, where the variable geometrical configuration of the experiment opens up to the possibility of collecting diffraction intensities along reciprocal space Bragg rods (B. Pedrini et al., Phil. Trans. R. Soc. B 369, 2014). The main bottleneck resides to date in $2 \mathrm{D}$ data processing and reduction due to the absence of dedicated software in conventional X-ray crystallography software suites.

The most recent advancements in 2D crystallography data treatment will be presented with particular focus on the opportunities and challenges of using intensities collected along reciprocal space rods.

Keywords: 2D-crystallography, membrane proteins, FEL

\section{MS3-P3 FIP-BM30A at the ESRF: an automated beamline for protein crystallography with unique features}

Jean-Luc Ferrer ${ }^{1}$, Yoann Sallaz-Damaz ${ }^{1}$, Xavier Vernede ${ }^{1}$, Michel Pirocchi $^{1}$, Christophe Berzin ${ }^{1}$, Monika Budayova-Spano ${ }^{1}$, Pascale Israel-Gouy ${ }^{1}$, Franck Borel $^{1}$, David Cobessi ${ }^{1}$

1. Institut de Biologie Structurale, Grenoble, France

email: jean-luc.ferrer@ibs.fr

FIP-BM30A is an automated beamline for protein crystallography at the ESRF. But it is also a very flexible beamline, that can host a large variety of experiments, such as on-line absorption spectroscopy, humidity controlled, micro-ship in situ diffraction, Etc.

From the seminal work accomplished on beamline FIP-BM30A (ESRF) in 2000' to the present developments, robot based systems significantly changed the crystallography experiment strategy. They open possibilities for new strategies, give a high flexibility to the experimental setup, and make automation and remote control much easier. The robotized platform in operation on FIP-BM30A, named G-Rob, plays as a fully integrated, multi-purpose automated and remotely controlled diffractometer. G-Rob integrates several functions: classical sample changer; goniometer for frozen samples or capillaries [1], including frozen sample transfer from a storage Dewar; crystallization trays handling for in situ screening and data collection on crystallization plates and microchips [2]; beam monitoring; on line crystal fluorescence/absorption; crystal harvesting; Etc. Thanks to its tool changer, the robot arm can go automatically from one application to another. G-Rob can be easily upgraded with new functions. As an example of this flexibility, the G-Rob system on FIP-BM30A was recently expended with a new functionality: a dual gripper for SPINE-format sample holder, that makes possible the sample dismounting/mounting operation in a single trajectory, reducing this way the cycle time for sample exchange by a factor $\sim 2$.

Among the last results obtained with G-Rob are: (i) Automated structure resolution at room temperature (in situ), for the analysis of protein dynamic; (ii) Automated structural screening for the fragment based drug design strategy. New functions are also under development, such as the remote controlled robotized crystal harvesting [3].

Another, but important, feature of beamline FIP-BM30A is a new web-based user interface, named WIFIP. With this interface, several users can share the control of the experiment, from sample handling to data reduction, through a web browser, on the beamline or from the lab or home. Web-based technology makes the access easy, and the communication very fluid, even with a limited bandwidth.

[1] Jacquamet et al., Acta Cryst. D, 2004, 60, 888-894.

[2] Jacquamet et al., Structure, 2004, 12, 1219-1225.

[3] Heidari Khajepour et al., Acta D, 2013, 69, 381-387. 


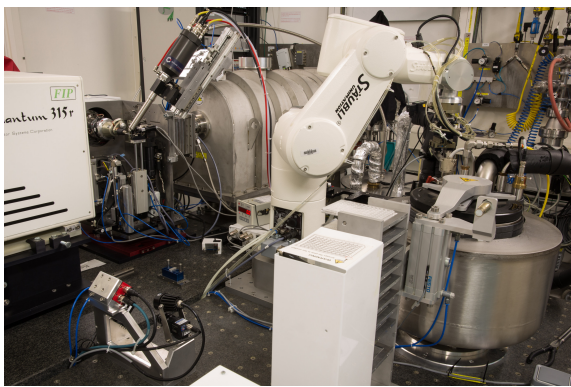

Figure 1. Overview of the robotized setup on beamline FIP-BM30A at the ESRF.

Keywords: beamline, macromolecular crystallography, automation, robot-based, crystallization, harvesting
MS3-P4 Improved beam delivery and new data collection tools on the macromolecular crystallography beamline I04 at Diamond Light Source

Ralf Flaig ${ }^{1}$, Pierpaolo Romano ${ }^{1}$, Jonathan Blakes ${ }^{1}$, Chris Bloomer ${ }^{1}$, Graham Duller $^{1}$, Sandira Gayadeen ${ }^{1}$, Michael Hillman ${ }^{1}$, James O'Hea ${ }^{1}$, Geoff Preece ${ }^{1}$, Graeme Winter ${ }^{1}$, David R. Hall ${ }^{1}$

1. Diamond Light Source, Harwell Science and Innovation Campus, Didcot, UK, OX11 0DE

email: ralf.flaig@diamond.ac.uk

Diamond Light Source [1] currently operates five beamlines for macromolecular crystallography (MX) and soon seven beamlines will serve the MX user community [2]. I04, a widely tuneable $(5-25 \mathrm{keV}$, core range 6-17 $\mathrm{keV}) \mathrm{SAD} / \mathrm{MAD}$ station [3] is constantly evolving with the aim to provide the user with state of the art tools for data collection, especially to enable structure solution from increasingly difficult and challenging samples. Since early 2015 the beamline is using an array of compound refractive lenses in a transfocator-like device allowing the user to change the beam size from 10 (h) $x 5$ (v) up to 110 (h) x 100 (v) microns over a wide energy range and thus be better able to match the beam size to the crystal size or be able to select more precisely the best part of the crystal. Using these small beam sizes, especially with small sample sizes, requires much more stringent schemes in terms of reliable beam delivery with respect to beam intensity and position than were envisaged for the original beamline design, including the monochromator. In addition, reliable beam diagnostics to monitor beam intensity and position over a wide beam size and energy range were not available at the beamline. We therefore have started a programme to improve beam delivery and have recently installed a new double crystal monochromator (DCM) which was designed inhouse based on existing proven technology and experience gained. The aim is to provide a system that is more stable, also taking into account possible future changes e.g. an increase in ring current, a lower emittance storage ring lattice and a new insertion device with higher power output. Alongside this, we have installed new beam position and intensity monitors that we will use in conjunction with a beam delivery feedback system. We are also looking into an improved goniometry and sample test system. As part of this we are planning the installation of the SmarGon multi-axis goniometer, which will enable better and faster sample centring, improved helical scan performance and provide us with new scientific capabilities for multi-sweep data collection strategies using multiple crystal orientations. Alongside these upgrades in beamline hardware we are also working on the development of the user software interface in order to streamline the user experiments.

References

[1] http://www.diamond.ac.uk

[2] http://www.diamond.ac.uk/Mx.html

[3] http://www.diamond.ac.uk/Beamlines/Mx/I04.html

Keywords: Diamond Light Source, macromolecular crystallography, beamlines, experimental phasing, data collection, mult-axis goniometry, monochromator, beam diagnostics, feedback 